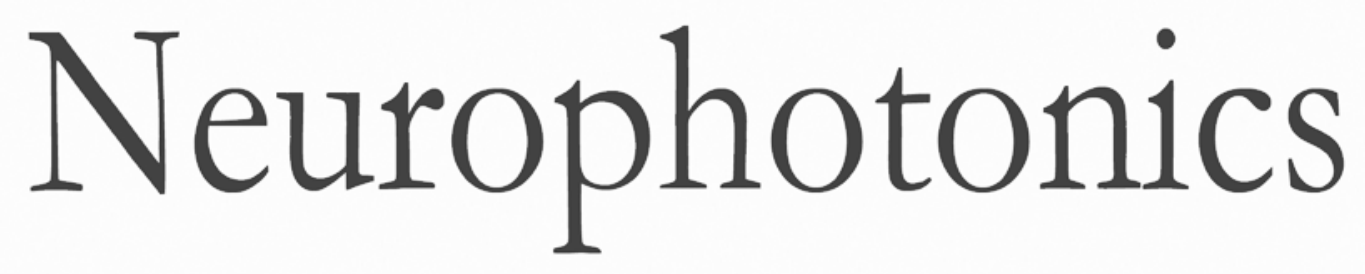

Neurophotonics.SPIEDigitalLibrary.org

\title{
Pioneers in Neurophotonics: Special Section Honoring Professor Lawrence B. Cohen
}

Brian M. Salzberg

Dejan Zecevic

\section{SPIE.}




\title{
Pioneers in Neurophotonics: Special Section Honoring Professor Lawrence B. Cohen
}

\author{
Brian M. Salzberg \\ University of Pennsylvania \\ Perelman School of Medicine \\ Philadelphia, Pennsylvania 19104, United States \\ E-mail: bmsalzbe@mail.med.upenn.edu \\ Dejan Zecevic \\ Yale University School of Medicine \\ New Haven, Connecticut 06510, United States \\ E-mail: dejan.zecevic@yale.edu
}

Larry Cohen began to explore optical approaches to membrane function (light scattering and birefringence) in the late 1960 s when he was still a postdoctoral fellow in the laboratory of Richard D. Keynes at the University of Cambridge. During the next forty years, Cohen became the dominant figure in developing and applying optical methods in cell physiology, biophysics, and neuroscience. Indeed, most of the myriad current techniques involving photonic measurements can be traced to Cohen's direct initiation or his active involvement. Beginning in the early 1970s, Larry Cohen began using voltage-clamped squid giant axons to screen literally thousands of substances, first fluorescent, and then merely colored, for their voltage sensitivity and freedom from phototoxicity. In 1972, Lawrence Cohen and collaborators showed that Merocyanine 540, the first sensitive molecular indicator of membrane potential (potentiometric dye), could be used to record the action potential, without averaging, from squid giant axons. In 1973, again using Merocyanine 540, Cohen and colleagues monitored electrical activity from an individual leech sensory neuron, and this was the first optical recording from a real intact neuron. Then, in 1977, Cohen's lab showed that as many as fourteen barnacle neurons could be monitored simultaneously, and individually, using voltage-sensitive absorbance dyes. Action potentials and synaptic potentials, both excitatory and inhibitory, could be recorded for many seconds. This began the era of optical recording from many neurons simultaneously, culminating in the recording of hundreds of neurons from preparations as diverse as invertebrate ganglia and mammalian cortices. Among many highlights from this period was the demonstration that simple learning paradigms in invertebrates involved the participation of hundreds of neurons. In nearly four decades, several thousand plausibly potentiometric probes have been synthesized and assayed, and, in the last decade, Larry has been in the forefront of exploring genetically encoded voltage-sensitive proteins. The vast majority of these molecules absorb and emit in the visible region of the spectrum and many have found wide application in studies of cell and tissue physiology. Now, the spectral range of voltage-sensitive dyes has been extended far into the red and will soon be extended into the infra-red. Not only will these molecular probes permit their use in tissues like the retina that are sensitive to photons in the visible, but longer wavelength excitation and emission will

C 2015 Society of Photo-Optical Instrumentation Engineers (SPIE) enable enhanced light penetration in three-dimensional structures like the heart and brain, where the spatiotemporal patterns of activity are neither simple nor always superficial. Among the earliest suggested applications of voltage-sensitive dyes was their potential use in probing membrane voltage at otherwise inaccessible regions of cells, perhaps best exemplified by the transverse tubular system of skeletal muscle. These were quickly realized, as were many more uses for these molecular voltmeters. From intramolecular profiling of the dynamic electric field to cortical and subcortical mapping of brain activity, Prof. Cohen's work and influence on optical recording of membrane potential in particular and optical methods in cell physiology in general have enlightened us all.

More personally, Larry has a remarkable quickness of mind and analytical ability. This is often belied by his slowness of speech, which usually reflects consideration and caution. He also evinces unusual talent for recognizing important outstanding problems in his area of interest, and in other areas as well. As a result, he is usually far in advance of his field, as has been the case with organic dye indicators and genetically encoded voltage sensors. Despite his numerous achievements and his distinction, Larry has remained a thoroughly modest and unassuming man. He has also been an exceptional mentor to scores of students, postdocs, and collaborators, many of whom went on to distinguished careers in several areas related to light and imaging.

From the earliest reports of optical probes of membrane potential, from Cohen's laboratory, ambitious ideas for the application of these molecular indicators to the study of the central nervous system were never far from Larry's consciousness. The tantalizing metaphor popularized by Sherrington, the "enchanted loom where millions of flashing shuttles weave a dissolving pattern, always a meaningful pattern though never an abiding one; a shifting harmony of subpatterns," drove scientists, led by Larry Cohen, to learn more about the brain, that "great raveled knot." The exploration of the central nervous system, much abetted by optical methods, is barely in its infancy. Already, many new observations have been reported, and many more are undoubtedly to come. These observations, barely conceivable without the functional optical mapping using potentiometric probes, and now, genetically encoded voltage-sensitive proteins, pioneered by Prof. Cohen and his colleagues, are only beginning. Indeed, the era in which optical methods, especially fluorescence, but also absorbance, birefringence, and light scattering, are applied to an extremely large range of biological preparations, could not have occurred without Larry Cohen's pioneering and extremely fruitful work. 